PROCEEDINGS OF THE

AMERICAN MATHEMATICAL SOCIETY

Volume 136, Number 12, December 2008, Pages 4143-4149

S 0002-9939(08)09434-3

Article electronically published on June 17, 2008

\title{
A SHORT PROOF OF THE MOCK THETA CONJECTURES USING MAASS FORMS
}

\author{
AMANDA FOLSOM
}

(Communicated by Ken Ono)

\begin{abstract}
A celebrated work of D. Hickerson gives a proof of the Mock Theta Conjectures using Hecke-type identities discovered by G. Andrews. Here, we respond to a remark by K. Bringmann, K. Ono and R. Rhoades and provide a short proof of the Mock Theta Conjectures by realizing each side of the identities as the holomorphic projection of a harmonic weak Maass form.
\end{abstract}

\section{INTRODUCTION}

Throughout we assume $\tau \in \mathbb{H}$, and let $q=q(\tau):=e^{2 \pi i \tau}, e(\alpha):=e^{2 \pi i \alpha}$. Ramanujan's "Lost" Notebook, discovered by George Andrews in 1976, contains a number of $q$-series identities involving Ramanujan's 17 original "mock theta" functions, which are classified by "order" 3,5 or 7 . In [2], the authors show that the ten $5^{\text {th }}$ order identities naturally divide into two classes and prove that any given identity is true if and only if all identities are true for every member of its class. Thus, the truth of all $5^{\text {th }}$ order identities reduces to the truth of two identities, which are called in 2 2 the "Mock Theta Conjectures". Before stating the Mock Theta Conjectures, we introduce the functions involved. We do so using the original notation of G. N. Watson (15], 16]), who proved claims stated about these mock theta functions in Ramanujan's last letter to G. H. Hardy [13]. As in [16] we define

$$
\begin{aligned}
\chi_{0}(q) & :=\sum_{n \geq 0} \frac{q^{n^{2}+n}}{\left(q^{n+1} ; q\right)_{n}}, \\
\chi_{1}(q) & :=\sum_{n \geq 0} \frac{q^{n}}{\left(q^{n+1} ; q\right)_{n+1}},
\end{aligned}
$$

where $(a ; q)_{n}:=\prod_{j=0}^{n-1}\left(1-a q^{j}\right)$. The Mock Theta Conjectures relate the functions $\chi_{0}(q)$ and $\chi_{1}(q)$ to differences of "rank generating functions"

$$
R_{b, c}(d ; q):=\sum_{n \geq 0}(N(b, 5,5 n+d)-N(c, 5,5 n+d)) q^{n},
$$

Received by the editors November 5, 2007.

2000 Mathematics Subject Classification. Primary 11F37.

The author is grateful for a National Science Foundation Postdoctoral Fellowship and wishes to thank Ken Ono for suggesting this project. The author also thanks the referee for a very detailed and thoughtful report, including useful suggestions that have helped ease the exposition of this paper.

(C)2008 American Mathematical Society 
where $N(b, t, r)$ denotes the number of partitions of $r$ with rank congruent to $b$ mod $t$. The rank of a given partition is a statistic defined by F.J. Dyson [9] as the number of parts of the partition subtracted from the largest part of the partition. (For example, the partition $1+1+1+3+4$ of 10 has rank equal to $4-5=-1$.)

Conjecture (The Mock Theta Conjecture:11).

$$
\begin{aligned}
\chi_{0}(q)-1 & =R_{1,0}(0 ; q), \\
\chi_{1}(q) & =R_{2,1}(3 ; q)+R_{2,0}(3 ; q) .
\end{aligned}
$$

\section{Proof of the Mock Theta Conjectures using Maass forms}

D. Hickerson, in the celebrated work [10, proves the Mock Theta Conjectures using Hecke-type identities discovered by G. Andrews [1]. More recently, in [6], the authors explicitly construct families of modular forms after offering a systematic treatment of more general mock theta-type identities. As a side remark to their results, [6, §1 Remark 2], the authors state:

...the results of Zwegers' thesis ... [and] the proof of [6. Thm. 1.1] ... reduces the proof of the Mock Theta Conjectures to the verification of two simple identities for classical weakly holomorphic modular forms.

Our purpose here in this paper is to respond to this remark and provide a proof of the Mock Theta Conjectures by realizing each side of the identities as the holomorphic projection of a harmonic weak Maass form. Until recently (as exposed in [17] and [4), not much was known regarding the role of Ramaunjan's mock theta functions within the context of modular forms. S. Zwegers "completed" many of Ramanujan's mock theta functions to obtain weight $1 / 2$ weak Maass forms, and in [4] K. Bringmann and K. Ono further generalize the results in 17]. (For the definition of weak Maass forms, see [8] or [4]-6].) We make use of these results to prove the Mock Theta Conjectures.

For a vector valued function $V(\tau):=\left(v_{1}(\tau), v_{2}(\tau), \ldots, v_{n}(\tau)\right)^{T}$, we let $V^{(i)}(\tau):=$ $v_{i}(\tau)$ denote the $i^{\text {th }}$ component function. We use the following identities of Watson [16:

$$
\begin{aligned}
& \chi_{0}(q)=2 F_{0}(q)-\phi_{0}(-q), \\
& \chi_{1}(q)=2 F_{1}(q)+q^{-1} \phi_{1}(-q),
\end{aligned}
$$

where $F_{j}(q)$ and $\phi_{j}(q), j=0,1$, are additional fifth order mock theta functions whose definitions ( $q$-series expansions) may be found in [2], for example.

Using the vectors $F_{5,1}(\tau)$ and $F_{5,2}(\tau)$ defined in [17, p. 74 and p. 79], we have

$$
\begin{aligned}
q^{\frac{1}{240}} F_{5,1}^{(3)}(\tau)+1 & =F_{0}\left(q^{\frac{1}{2}}\right), & q^{\frac{1}{240}} F_{5,2}^{(3)}(\tau) & =\phi_{0}\left(-q^{\frac{1}{2}}\right), \\
q^{-\frac{71}{240}} F_{5,1}^{(4)}(\tau) & =F_{1}\left(q^{\frac{1}{2}}\right), & -q^{\frac{49}{240}} F_{5,2}^{(4)}(\tau) & =\phi_{1}\left(-q^{\frac{1}{2}}\right),
\end{aligned}
$$

\footnotetext{
${ }^{1}$ We point out that the second Mock Theta Conjecture stated here is corrected from [2, Eq. (4.10)], where an extra " -1 " appears. This extraneous " -1 " first appears in [2, Eq. (4.7)], which one confirms is merely miscopied from 3 Eq. (6.18)].
} 
so that

$$
\begin{gathered}
\chi_{0}\left(q^{\frac{1}{2}}\right)-1=1+q^{\frac{1}{240}}\left(2 F_{5,1}^{(3)}(\tau)-F_{5,2}^{(3)}(\tau)\right), \\
\chi_{1}\left(q^{\frac{1}{2}}\right)=q^{-\frac{71}{240}}\left(2 F_{5,1}^{(4)}(\tau)-F_{5,2}^{(4)}(\tau)\right) .
\end{gathered}
$$

We define the differences

$$
\begin{aligned}
& K_{1}(\tau):=q^{-\frac{1}{240}}\left(\chi_{0}\left(q^{\frac{1}{2}}\right)-1\right)-2 G_{5,1}^{(3)}(\tau)+G_{5,2}^{(3)}(\tau)-q^{-\frac{1}{240}}, \\
& N_{1}(\tau):=q^{\frac{71}{240}}\left(\chi_{1}\left(q^{\frac{1}{2}}\right)-2 G_{5,1}^{(4)}(\tau)+G_{5,2}^{(4)}(\tau)\right)
\end{aligned}
$$

and find

$$
\begin{aligned}
& K_{1}(\tau)=2 H_{5,1}^{(3)}(\tau)-H_{5,2}^{(3)}(\tau), \\
& N_{1}(\tau)=2 H_{5,1}^{(4)}(\tau)-H_{5,2}^{(4)}(\tau),
\end{aligned}
$$

where the vectors $G_{5, j}(\tau)$ and $H_{5, j}(\tau), j=1,2$, are defined in [17. Using (2.1), (2.2), and [17, Prop. 4.10, Prop. 4.13, Prop. 4.2 (2)], we deduce the following proposition.

Proposition 2.1. The functions $K_{1}(\tau)$ and $N_{1}(\tau)$ are vector valued harmonic weak Maass forms of weight $1 / 2$, whose non-holomorphic parts $K_{1}^{\mathrm{nh}}(\tau)$ and $N_{1}^{\mathrm{nh}}(\tau)$ of $K_{1}(\tau)$ and $N_{1}(\tau)$ (respectively) are given by

$$
\begin{aligned}
& K_{1}^{\mathrm{nh}}(\tau)=-\frac{3 i}{4 \sqrt{30}} \int_{-\bar{\tau}}^{i \infty} \frac{\sum_{m \equiv 19,29 \bmod 60} m e^{\pi i m^{2} z / 120}-\sum_{m \equiv 49,59 \bmod 60} m e^{\pi i m^{2} z / 120}}{\sqrt{-i(z+\tau)}} d z, \\
& N_{1}^{\mathrm{nh}}(\tau)=-\frac{3 i}{4 \sqrt{30}} \int_{-\bar{\tau}}^{i \infty} \frac{\sum_{m \equiv 13,23 \bmod 60} m e^{\pi i m^{2} z / 120}-\sum_{m \equiv 43,53 \bmod 60} m e^{\pi i m^{2} z / 120}}{\sqrt{-i(z+\tau)}} d z .
\end{aligned}
$$

Next we define the function: 2

$$
\begin{aligned}
\widetilde{K}_{2}(\tau) & :=q^{-\widetilde{\ell_{5}}} R_{1,0}\left(0, q^{5 \ell_{5}}\right)-q^{-\widetilde{\ell_{5}}} \\
& =\sum_{n \geq 0}(N(1,5,5 n)-N(0,5,5 n)) q^{5 \ell_{5} n-\widetilde{\ell}_{5}}-q^{-\widetilde{\ell_{5}}}, \\
\widetilde{N}_{2}(\tau) & :=q^{3 \ell_{5}-\widetilde{\ell}_{5}}\left(R_{2,1}\left(3 ; q^{5 \ell_{5}}\right)+R_{2,0}\left(3 ; q^{5 \ell_{5}}\right)\right) \\
& =\sum_{n \geq 0}(2 N(2,5,5 n+3)-N(1,5,5 n+3)-N(0,5,5 n+3)) q^{\ell_{5}(5 n+3)-\widetilde{\ell}_{5}},
\end{aligned}
$$

where throughout we assume the notation as in [4] and let $\ell_{5}:=25 \cdot 24$, and $\tilde{\ell}_{5}:=25$. We deduce from [4, Theorem 1.3] that $\widetilde{K}_{2}(\tau)$ and $\widetilde{N}_{2}(\tau)$ are restricted sums of the holomorphic parts of harmonic weak Maass forms. To make this statement more precise, as introduced in 4 , we define $S_{1}\left(\frac{\alpha}{5}, \tau\right)$ by

$$
S_{1}\left(\frac{\alpha}{5}, \tau\right):=-i \sin \left(\frac{\pi \alpha}{5}\right) 10 \sqrt{2} \int_{-\bar{\tau}}^{i \infty} \frac{\Theta\left(\frac{\alpha}{5} ; 5^{2} \cdot 24 \cdot z\right)}{\sqrt{-i(\tau+z)}} d z
$$

\footnotetext{
${ }^{2}$ Different definitions for $N(0, t, 0)$ exist in the literature, e.g. $N(0, t, 0):=0$ in [2], 3], while in other places (e.g. 4], 6]), $N(0, t, 0):=1$. We adopt the former convention, therefore requiring the extra term $-q^{-\widehat{\ell}_{5}}$ in the definition of $\widetilde{K}_{2}(\tau)$. (See also Proposition 2.2 )
} 
where

$$
\Theta\left(\frac{\alpha}{5} ; \tau\right):=\sum_{m \bmod 10}(-1)^{m} \sin \left(\frac{\alpha \pi(6 m+1)}{5}\right) \sum_{n \equiv 6 m+1 \bmod 60} n e\left(n^{2} \tau / 24\right) .
$$

After a brief argument (see also [4, Prop. 4.1]), one finds that the function $S_{1}\left(\frac{\alpha}{5}, \tau\right)$ has Fourier development of the form

$$
S_{1}\left(\frac{\alpha}{5}, \tau\right)=\sum_{\substack{m \\ n \equiv 6 m+1 \bmod 60}} \beta_{n, m} q^{-(5 n)^{2}}
$$

We define $S_{1}^{*}\left(\frac{\alpha}{5} ; \tau\right)$ (resp. $\left.S_{1}^{* *}\left(\frac{\alpha}{5} ; \tau\right)\right)$ to be the restriction of (the Fourier development of) $S_{1}\left(\frac{\alpha}{5} ; \tau\right)$ to those $n$ such that $n^{2} \equiv 1 \bmod 120$ (respectively $n^{2} \equiv 49$ mod 120). Finally, we define

$$
\begin{aligned}
& K_{2}(\tau):=\widetilde{K}_{2}(\tau)-\frac{1}{5} \sum_{j=1}^{4}\left(\zeta_{5}^{-j}-1\right) S_{1}^{*}\left(\frac{j}{5} ; \tau\right), \\
& N_{2}(\tau):=\widetilde{N}_{2}(\tau)-\frac{1}{5} \sum_{j=1}^{4}\left(2 \zeta_{5}^{-2 j}-\zeta_{5}^{-j}-1\right) S_{1}^{* *}\left(\frac{j}{5} ; \tau\right),
\end{aligned}
$$

where $\zeta_{5}:=e^{2 \pi i / 5}$. We deduce the following proposition.

Proposition 2.2. The functions $K_{2}(\tau)$ and $N_{2}(\tau)$ are harmonic weak Maass forms of weight $\frac{1}{2}$ on $\Gamma_{1}\left(144 \cdot 10^{2} \cdot 5^{4}\right)$.

Proof. By Theorem 1.3 in [4, for $0 \leq r<5$, the difference $\Theta^{3}$

$$
\sum_{n \geq 0}\left(N(r, 5 ; n)-\frac{p(n)}{5}\right) q^{24 \cdot 5^{2} \cdot n-5^{2}}+\lambda_{r}
$$

is the holomorphic part of a weak Maass form of weight $\frac{1}{2}$ on $\Gamma_{1}\left(144 \cdot 10^{2} \cdot 5^{2}\right)$, where $p(n)$ denotes the number of partitions of $n$, and $\lambda_{r}:=0$ for $r \neq 0$, and $\lambda_{0}:=q^{-25}$. Turning to the proof of this statement [4, $\S 3.3$, p. 24], we find that the non-holomorphic part is given by $-\frac{1}{5} \sum_{j=1}^{4} \zeta_{5}^{-r j} S_{1}\left(\frac{j}{t} ; \tau\right)$. For $0 \leq j \leq 4$ we define

$$
\begin{aligned}
\mathfrak{D}_{j}(\tau):= & \sum_{n \geq 0}\left(N(r, 5 ; n)-\frac{p(n)}{5}\right) q^{24 \cdot 5^{2} \cdot n-5^{2}}-\frac{1}{5} \sum_{j=1}^{4} \zeta_{5}^{-r j} S_{1}\left(\frac{j}{t} ; \tau\right)+\lambda_{j}, \\
\mathfrak{D}_{j}^{*}(\tau):= & \sum_{n \geq 0}\left(N(r, 5 ; 5 n)-\frac{p(5 n)}{5}\right) q^{24 \cdot 5^{2} \cdot 5 n-5^{2}}-\frac{1}{5} \sum_{j=1}^{4} \zeta_{5}^{-r j} S_{1}^{*}\left(\frac{j}{t} ; \tau\right)+\lambda_{j}, \\
\mathfrak{D}_{j}^{* *}(\tau):= & \sum_{n \geq 0}\left(N(r, 5 ; 5 n+3)-\frac{p(5 n+3)}{5}\right) q^{24 \cdot 5^{2} \cdot(5 n+3)-5^{2}} \\
& \quad-\frac{1}{5} \sum_{j=1}^{4} \zeta_{5}^{-r j} S_{1}^{* *}\left(\frac{j}{t} ; \tau\right) .
\end{aligned}
$$

\footnotetext{
${ }^{3}$ The factor $q^{-25}$ must be introduced when $r=0$, as we have adopted the convention $N(0, t, 0):=0$ as in [2], 3], whereas $N(0, t, 0):=1$ in [4].
} 
Then one finds

$$
\begin{aligned}
& K_{2}(\tau)=\mathfrak{D}_{1}^{*}(\tau)-\mathfrak{D}_{0}^{*}(\tau), \\
& N_{2}(\tau)=2 \mathfrak{D}_{2}^{* *}(\tau)-\mathfrak{D}_{1}^{* *}(\tau)-\mathfrak{D}_{0}^{* *}(\tau) .
\end{aligned}
$$

By [4, the forms $\mathfrak{D}_{j}(\tau)$ are weight $\frac{1}{2}$ harmonic weak Maass forms on $\Gamma_{1}(144$. $\left.10^{2} \cdot 5^{2}\right)$. The forms $\mathfrak{D}_{j}^{*}(\tau)$ and $\mathfrak{D}_{j}^{* *}(\tau)$ are restrictions of $\mathfrak{D}_{j}(\tau)$ to the arithmetic progressions $n \equiv 0 \bmod 5$ and $n \equiv 3 \bmod 5$ and thus, too, are harmonic weak Maass forms by a classical argument using the orthogonality of Dirichlet characters (11], Proposition 17) 4 After restriction, the group $\Gamma_{1}\left(144 \cdot 10^{2} \cdot 5^{2}\right)$ becomes $\Gamma_{1}\left(144 \cdot 10^{2} \cdot 5^{4}\right)$. Proposition 2.2 now follows from (2.3) and (2.4).

Next we explicitly compute the non-holomorphic part $K_{2}^{\mathrm{nh}}(\tau)\left(\right.$ resp. $\left.N_{2}^{\mathrm{nh}}(\tau)\right)$ of $K_{2}(\tau)$ (resp. $N_{2}(\tau)$ ). We first consider $K_{2}(\tau)$. By definition, the Fourier expansion of $S_{1}^{*}(\alpha, \tau)$ reduces to a sum over those integers $n$ for which $n^{2} \equiv 1 \bmod 120$. The integers $n$ satisfying this condition are

$$
n \in \Omega:=\{1,11,19,29,31,41,49,51,61,71,79,89,91,101,109,119\}(\bmod 120) .
$$

Further, of all residue classes $m \bmod 10$, we find there are only 8 values for $n \in \Omega$ that satisfy $n \equiv 6 m+1 \bmod 60$, and these are

$$
n \in \Omega^{\prime}:=\{1,19,31,49,61,79,91,109\}(\bmod 120),
$$

corresponding to, in order,

$$
m \in \Psi:=\{0,3,5,8,10,13,15,18\} .
$$

We observe that

$$
\Omega^{\prime}(\bmod 60)=\{19,-29,49,-59\}(\bmod 60)
$$

and find

$$
\begin{aligned}
K_{2}{ }^{\mathrm{nh}}(\tau)=-\frac{15 i}{\sqrt{2}}\left(\sum_{n \equiv 19,29 \bmod 60} \int_{-\bar{\tau}}^{i \infty} \frac{n e^{2 \pi i(5 n)^{2} z}}{\sqrt{-i(\tau+z)}} d z\right. & \\
& \left.\quad-\sum_{n \equiv 49,59 \bmod 60} \int_{-\bar{\tau}}^{i \infty} \frac{n e^{2 \pi i(5 n)^{2} z}}{\sqrt{-i(\tau+z)}} d z\right) .
\end{aligned}
$$

Letting $\tau \mapsto 10 \ell_{5} \tau$ in Proposition 2.1, we find

$$
\begin{aligned}
K_{1}^{\mathrm{nh}}\left(10 \ell_{5} \tau\right) & =-\frac{15 i}{\sqrt{2}} \int_{-\bar{\tau}}^{i \infty} \frac{\sum_{m \equiv 19,29 \bmod 60} m e^{2 \pi i(5 m)^{2} z}-\sum_{m \equiv 49,59 \bmod 60} m e^{2 \pi i(5 m)^{2} z}}{\sqrt{-i(z+\tau)}} d z \\
& =K_{2}{ }^{\mathrm{nh}}(\tau) .
\end{aligned}
$$

\footnotetext{
${ }^{4}$ To be more precise, for those readers who wish to carry out the details of this assertion, one obtains such restricted forms by applying the Hecke operators $U$ and $V$, and twisting. For example, if $g(\tau):=\sum_{n} a_{n} q^{5^{2}(24 n-1)}$, then

$$
g^{*}(\tau):=\sum_{n \equiv 0 \bmod 5} a_{n} q^{5^{2}(24 n-1)}=\frac{1}{4} \sum_{\chi \bmod 5}\left(\chi(4)\left(g \mid U_{25}\right) \otimes \bar{\chi} \mid V_{25}\right) .
$$
}


For the functions $N_{2}(\tau)$, we proceed similarly, using the sets

$$
\begin{aligned}
\Psi_{2} & :=\{1,2,6,7\}(\bmod 10), \\
\Omega_{2}^{\prime}(\bmod 60) & :=\{13,-23,43,-53\}(\bmod 60),
\end{aligned}
$$

and conclude $N_{1}^{\mathrm{nh}}\left(10 \ell_{5} \tau\right)=N_{2}^{\mathrm{nh}}(\tau)$.

To conclude the proof of the Mock Theta Conjectures, by Proposition 2.2 we have that $K_{2}(\tau)$ and $N_{2}(\tau)$ are harmonic weak Maass forms of weight $\frac{1}{2}$ on $\Gamma_{1}\left(144 \cdot 10^{2}\right.$. $\left.5^{4}\right)$. The forms $K_{1}\left(10 \ell_{5} \tau\right)$ and $N_{1}\left(10 \ell_{5} \tau\right)$ are also harmonic weak Maass forms of weight $\frac{1}{2}$ by Proposition 2.1. We remark that the groups on which all forms $K_{1}(\tau)$, $K_{2}(\tau), N_{1}(\tau)$ and $N_{2}(\tau)$ live are dictated by the groups associated to the theta functions appearing in their respective period integrals 5 By a lifting argument as in 8 and as used in [4, one finds an optimal (larger) subgroup associated to $K_{1}\left(10 \ell_{5} \tau\right)$ and $N_{1}\left(10 \ell_{5} \tau\right)$, namely $\Gamma_{1}\left(144 \cdot 10^{2} \cdot 5^{4}\right)$. For brevity, we proceed by arguing the proof of the first Mock Theta Conjecture, as the second follows similarly. By (2.5), we see that the non-holomorphic parts of the forms $K_{1}\left(10 \ell_{5} \tau\right)$ and $K_{2}(\tau)$ are identical, and thus $\mathfrak{K}(\tau):=K_{1}\left(10 \ell_{5} \tau\right)-K_{2}(\tau)$ is a weakly holomorphic modular form of weight $\frac{1}{2}$ on $\Gamma_{1}\left(144 \cdot 10^{2} \cdot 5^{4}\right)$. We argue that $\mathfrak{K}(\tau)=0$ and provide two arguments.

Argument 1. We first claim that the weakly holomorphic modular form $\mathfrak{K}(\tau)$ is in fact holomorphic. From [17] and [4], one has all transformation laws for $\mathfrak{K}(\tau)$. Moreover, one has vector-valued forms containing $\mathfrak{K}(\tau)$ (from the vectors $H_{5,1}(\tau)$ and $H_{5,2}(\tau)$ of [17, and [4, Lemma 3.1]). With this, and the fact that the principal parts and constant terms are equal, an application of the circle method implies that the asymptotic growth of the coefficients of $\mathfrak{K}(\tau)$ is $\mathcal{O}\left(n^{\alpha}\right)$ for some constant $\alpha$. Indeed, one may see this result carried out in explicit detail in [7, Thm. 1.1 and Thm. 1.2] for the forms $R_{b, c}(d ; q)$. (See also [5, Cor 4.2 and p. 22].) Thus, $\mathfrak{K}(\tau)$ is a holomorphic modular form of weight $\frac{1}{2}$ on $\Gamma_{1}\left(144 \cdot 10^{2} \cdot 5^{4}\right)$. By the Serre-Stark Basis Theorem [12, Thm. 1.45] and [12, Eq. (1.7)], one has as a basis for the space of weight $\frac{1}{2}$ modular forms a finite number of theta functions, and one verifies that $\mathfrak{K}(\tau)$ is identically zero.

Argument 2. Arguing using complex analysis, one has the well-known valence formula 6

$$
\sum_{\tau \in \mathbb{H}} \operatorname{ord}_{\tau} \mathfrak{K}+\sum_{\text {cusps } \kappa} \operatorname{ord}_{\kappa} \mathfrak{K}+\operatorname{ord}_{\infty} \mathfrak{K}=\frac{1}{24}\left[\mathrm{SL}_{2}(\mathbb{Z}): \Gamma_{1}\left(144 \cdot 10^{2} \cdot 5^{4}\right)\right] .
$$

A priori, $\mathfrak{K}(\tau)$ is holomorphic on $\mathbb{H}$, so the first summand in (2.6) is non-negative. Further, we are able to determine a bound $-M \leq 0$ such that $\operatorname{ord}_{\kappa} \mathfrak{K} \geq-M$ for any cusp $\kappa$. This integer $-M$ is at worst the largest (in magnitude) negative order at $\infty$ of any other component in the vector-valued form to which $\mathfrak{K}$ belongs. Thus, by computing sufficiently many coefficients in the Fourier expansion of $\mathfrak{K}$ at $\infty$, one can contradict equation (2.6). Although the number of coefficients that must be checked is large due to the large index $\left[\mathrm{SL}_{2}(\mathbb{Z}): \Gamma_{1}\left(144 \cdot 10^{2} \cdot 5^{4}\right)\right]$, this number is indeed finite.

\footnotetext{
${ }^{5}$ See for example [14 for the transformation laws of the theta functions.

${ }^{6}$ The notation "ord" denotes appropriately weighted orders.
} 


\section{REFERENCES}

[1] G. Andrews, The fifth and seventh order mock theta functions, Trans. Am. Math. Soc. 293 (1986), 113-134. MR814916 (87f:33011)

[2] G. Andrews and F. Garvan, Ramanujan's "Lost" Notebook IV: The Mock Theta Conjectures, Adv. Math. 73 (1989), 242-255. MR987276(90d:11115)

[3] A.O.L. Atkin and P. Swinnerton-Dyer, Some properties of partitions, Proc. London Math. Soc. (3) 4 (1954), 84-106. MR0060535 (15:685d)

[4] K. Bringmann and K. Ono, Dyson's ranks and Maass forms, Annals of Mathematics, accepted for publication (2007).

[5] K. Bringmann and K. Ono, The $f(q)$ mock theta function conjecture and partition ranks, Inventiones Math. 165 (2006), 243-266. MR2231957 (2007e:11127)

[6] K. Bringmann, K. Ono, and R. Rhoades, Eulerian series as modular forms, Journal of the Amer. Math. Soc., accepted for publication.

[7] K. Bringmann, Asymptotics for rank partition functions, Trans. Amer. Math. Soc., accepted for publication.

[8] J. H. Bruinier and J. Funke, On two geometric theta lifts, Duke Math. J. 125 (2004), 45-90. MR:2097357 (2005m:11089)

[9] F. J. Dyson, Some guesses in the theory of partitions, Eureka (Cambridge) 8 (1944), 10-15.

[10] D. Hickerson, A proof of the Mock Theta Conjectures, Inventiones Math. (3) 94 (1988), 639-660. MR969247 (90f:11028a)

[11] N. Koblitz, Introduction to Elliptic Curves and Modular Forms, GTM No. 97, Springer-Verlag (1984, 1993). MR.1216136 (94a:11078)

[12] K. Ono, The web of modularity: Arithmetic of the coefficients of modular forms and qseries, Conference Board of the Mathematical Sciences 102, Amer. Math. Soc., Providence, RI (2004). MR2020489 (2005c:11053)

[13] S. Ramanujan, Collected Papers, Cambridge Univ. Press, London/NY, 1927 (reprinted by Chelsea, NY). MR 2280843 (2008b:11002)

[14] G. Shimura, On modular forms of half integral weight, Ann. of Math. 97 (1973), 440-481. MR0332663 (48:10989)

[15] G. N. Watson, The final problem: An account of the mock theta functions, J. London Math. Soc. 11 (1936), 55-80. MR1862757

[16] G.N. Watson, The mock theta functions (2), Proc. London Math. Soc. (2) 42 (1937), 274-304.

[17] S. P. Zwegers, Mock theta functions, Ph.D. Thesis, Universiteit Utrecht (2002).

Department of Mathematics, University of Wisconsin, 480 Lincoln Drive, Madison, WISCONSIN 53706

E-mail address: folsom@math.wisc.edu 